\title{
Design Improvement of a Linear Control Solenoid Valve using Multiphysics Simulation
}

\author{
GEE-SOO LEE \\ Youngsan University, 50510, 288 Junam-ro, Yangsan-si, Gyeongnam, Republic of Korea, E-mail: gslee@ysu.ac.kr
}

cross $^{\text {ref }}$ http://dx.doi.org/10.5755/j01.mech.24.3.20137

\section{Introduction}

The linear control solenoid valve is an electromagnetically actuated control valve that controls a plunger stroke, according to the amount of current supplied from the controller to the solenoid coil. Since linearity is a key parameter in designing linear control solenoid valves, it is necessary to design them with a minimum number of dead zones where the valve does not move in response to the external current $[1,2]$. The spool stroke is determined by the balance of forces, which comprise electromagnetic force, damping force, spring force, and hydraulic force. In turn, the spool stroke works on these forces. As shown in Fig. 1, the electromagnetic field is therefore strongly coupled with a flow field. In the electromagnetic component, the geometry of a core pole and the number of coil turns directly affect the electromagnetic force in the line control solenoid valve. In the hydraulic component, linear control solenoid valves are characterized by the variation of the equivalent orifice area with the spool stroke.

Most previous studies have dealt with the electromagnetic and hydraulic performances on an individual basis. Davis and Stewart performed two-dimensional numerical simulations of a globe control valve [3] and conducted experiments to verify an axisymmetric numerical model of the control valve [4]. Yuan and Li [5] investigated the effects on the steady flow force of various material properties. Lee et al. [6] analyzed the effects of notch shape on the flow force. The notches were able to minimize the flow force of the spool for a compensated spool notch. Amirante et al. [7] performed three-dimensional numerical simulations of a closed center directional control valve. Xie [8] performed a system-level analysis to investigate the effect of flow forces on a flow control variable force solenoid used in automatic transmission. A force-balance analysis was conducted on the spool valve in the variable force solenoid, in order to study the relationships among the control current, flow forces, spring forces, and flow area. Lequesne [9] conducted the finite element analysis of a solenoid for the fluid flow control and analyzed the effect of the control cone to achieve the constant force characteristics. Arakawa and Niimi [10] introduced the optimization technology for a magnetic circuit, which can be used to effectively design a compact linear solenoid valve. Vaughan and Gamble [11] introduced the nonlinear dynamic model of a high speed direct acting solenoid valve, which consisted of a proportional solenoid and a spool assembly. The model accurately predicted both the dynamic and steady-state response of the valve. Pappalardo and Guda [12] introduced a new computational algorithm for the numerical solution of the adjoint equations for nonlinear optimal control problem. They conducted the re- duction of the mechanical vibrations of a nonlinear two degrees-of-freedom dynamical system.

Multiphysics simulation can lead to considerable success in the design and optimization of linear control solenoid valves. To obtain the linearity of the linear control solenoid valve, the effects of various design parameters on the dynamic behaviors of the valve should be analyzed by a multiphysics simulation coupled with a 1-DOF system. Recently, many industries have used multiphysics simulations of coupled electromagnetic and fluid fields. To design a high-performance fuel injector for motorcycle engines, Watanabe et al. [13] conducted dynamic electromagnetic field simulations. They optimized the geometry of the magnetic path and developed a new injector. To describe the dynamical motion of the injector, the hydraulic force acting on the needle valve was assumed to be constant, though no flow field simulations were conducted. Bircher and Marmet [14] performed multiphysics simulations of electromagnetic micro valves for automatic dispensing in chemistry applications. Naseradinmousavi and Nataraj [15] derived and solved the analytical equations for a butterfly valve, including electromagnetics, fluid mechanics, and mechanical dynamics, with butterfly valves being strongly nonlinear. The coupled models were derived by making some simplifying assumptions. The results showed that the behavior of hydrodynamic torque played an important role in the closing and opening processes. Lee et al. [16] introduced a multiphysics analysis of a linear control solenoid valve and analyzed the effects of varying the supplied pressure, rising time, and maximum external current on the behavior and pressure sensitivities of the linear control solenoid. Mutschler et al. [17] performed multiphysics network simulations for a solenoid dispensing valve. To investigate the physical effects, which were electro-magnetic, fluid flow and fluid structure interaction in a solenoid dispensing valve, one-dimensional network simulation method was employed. The lumped elements were linked together to form a complete model of the dispensing valve. This approach has led to considerable success in the design and optimization to obtain better performance.

The main objectives of the present study were to improve the design of the linear control solenoid valve via a mutiphysics simulation coupled with a 1-DOF (Degree of Freedom) system. The multiphysics modelling included Ampere's law and incompressible Navier-Stokes equation when there were moving objects involved. First, to ensure a constant electromagnetic force with plunger strokes, the electromagnetic component of the linear control solenoid valve was improved. The controllable range of the final model, which was modified by the hydraulic component and the system parameters, can precisely control the pressure 
during the external current flow. It became $68.3 \%$ of the total control range.

\section{A linear control solenoid valve}

Fig. 1 shows a three-dimensional modelling view and the operating sequences of a linear control solenoid valve used in a hydraulic control system. A linear control solenoid valve simply consists of electromagnetic and hydraulic components. An electromagnetic force is generated if a current is applied to the solenoid coil. At the same time, the pressure supplied by the supply port generates the hydraulic force that moves the spool in a forward direction. If the sum of the electromagnetic force and the hydraulic force is greater than the spring force, the spool begins to move. The spool strokes are mechanically determined by the balances of the forces [8]. The hydraulic force created by the feedback pressure, which is determined by the spool stroke, pushes the spool forward. Conversely, the spool strokes affect the electromagnetic force and the hydraulic force. When an electric current is removed from the coil, the spool (or the plunger) returns via the restoring force. The system includes a two-way coupling system by way of the electromagnetic and flow fields [16].

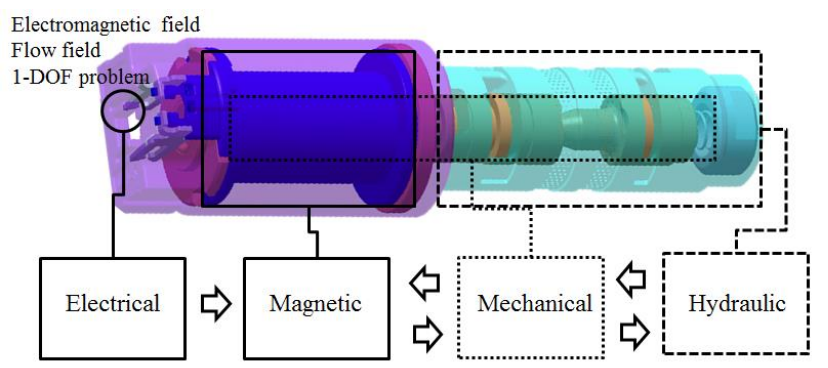

Fig. 13 -Dimensional modeling view and the operating sequences of a linear control solenoid valve

\section{Numerical method}

The linear control solenoid valve is comprised of two ordinary differential equations describing the electrical signal and Newton's 2nd law of motion, and of two partial differential equations, which are Ampere's law and the incompressible Navier-Stokes equation [16]. These equations are modelled and simulated using the commercial code, COMSOL 5.0, which is a cross-platform finite element analysis, solver and multiphysics software [18]. A transient analysis of Ampere's law, which is the axially symmetrical, was performed in order to simulate the electromagnetic field. By using the constitutive relation $\mathbf{B}=\mu_{0} \mu_{r} \mathbf{H}$ and the magnetic vector potential $\mathbf{B}=\nabla \times \mathbf{A}$, this law can be expressed as [19]:

$$
\sigma \frac{\partial \mathbf{A}}{\partial t}+\nabla \times\left(\frac{1}{\mu_{0} \mu_{r}} \nabla \times \mathbf{A}\right)=\mathbf{J}^{e},
$$

here: $B$ is the magnetic flux density, $\mathrm{T} ; \mathbf{H}$ is the magnetic field, $\mathrm{A} / \mathrm{m} ; \mathbf{J}^{e}$ is the externally generated current density vector, $\mathrm{A} / \mathrm{m}^{2}, \sigma$ is the electrical conductivity, $\mathrm{S} / \mathrm{m}, \mu_{r}$ is the relative magnetic permeability, and $\mu_{0}$ is the permeability of free space with $\mu_{0}=4 \pi \times 10^{-7} \mathrm{H} / \mathrm{m}$.
The governing equation for the flow inside the linear control solenoid valve is an axially symmetrical incompressible Navier-Stokes equation. The differential forms of the general conservations can be expressed as follows:

$$
\begin{aligned}
& \rho \nabla \cdot \mathbf{u}=0, \\
& \rho \frac{\partial \mathbf{u}}{\partial t}+\rho \mathbf{u} \cdot \nabla \mathbf{u}=-\nabla \mathbf{p}+\mu \nabla^{2} \mathbf{u},
\end{aligned}
$$

where: $\rho$ is the density of the working fluid, $\mathrm{kg} / \mathrm{m}^{3}, \mathbf{u}$ is the velocity of the flow, $\mathrm{m} / \mathrm{s}$, and $\mu$ is the dynamic viscosity of the fluid, $\mathrm{Pa} \cdot \mathrm{s}$.

In the single DOF mass-spring-damper system, Newton's $2^{\text {nd }}$ law of motion can be written as:

$$
\ddot{x}_{\mathrm{p}}+\frac{k_{\mathrm{s}}}{m_{\mathrm{t}}}\left(x_{\mathrm{p}}+x_{0}\right)+\frac{C}{m_{\mathrm{t}}}\left(\dot{x}_{\mathrm{p}}\right)-\frac{1}{m_{\mathrm{t}}}\left(F_{\mathrm{m}, \mathrm{z}}+F_{\mathrm{f}, \mathrm{z}}\right)=0,
$$

where: $m_{t}$ is the total mass of the plunger and the spool, $\mathrm{kg}$, $k_{s}$ is the spring constant, $\mathrm{N} / \mathrm{m}$, and $C$ is the damping coefficient, Ns/m. $F_{m, z}$ and $F_{f, z}$ are the z-directional forces, N acting on the plunger and the spool. The initial conditions of the spool are $x_{p, 0}=\dot{x}_{p, 0}=0$. Further details of the equations are provided in the references [16, 18].

Table 1 summarizes the boundary conditions and properties used in the multiphysics simulations. The properties of the fluid were set at values of the normal operation temperature of the automatic transmission fluid, $T_{s}=80^{\circ} \mathrm{C}$.

Table 1

Boundary conditions

\begin{tabular}{|c|c|c|c|}
\hline No. & Parameter & Unit & Value \\
\hline 1 & $i_{\max }$ & A & 1.2 \\
\hline 2 & $N_{p}$ & turns & 530 \\
\hline 3 & $\sigma_{\text {coil }}$ & $\mathrm{S} / \mathrm{m}$ & $6 \times 10^{7}$ \\
\hline 4 & $A_{\text {coil }}$ & $\mathrm{m}^{2}$ & $1 \times 10^{-6}$ \\
\hline 5 & $P_{s}$ & $\mathrm{bar}$ & 15 \\
\hline 6 & $P_{e}$ & $\mathrm{bar}$ & 0 \\
\hline 7 & $\rho$ & $\mathrm{kg} / \mathrm{m}^{3}$ & 852.07 \\
\hline 8 & $\mu$ & $\mathrm{Pa} \cdot \mathrm{s}$ & 0.03175 \\
\hline 9 & $m_{t}$ & $\mathrm{~kg}$ & 0.02839 \\
\hline 10 & $k_{s}$ & $\mathrm{~N} / \mathrm{m}$ & 1,794 \\
\hline 11 & $C$ & $\mathrm{~N} \cdot \mathrm{s} / \mathrm{m}$ & 6 \\
\hline 12 & $x_{0}$ & $\mathrm{~mm}$ & 6 \\
\hline
\end{tabular}

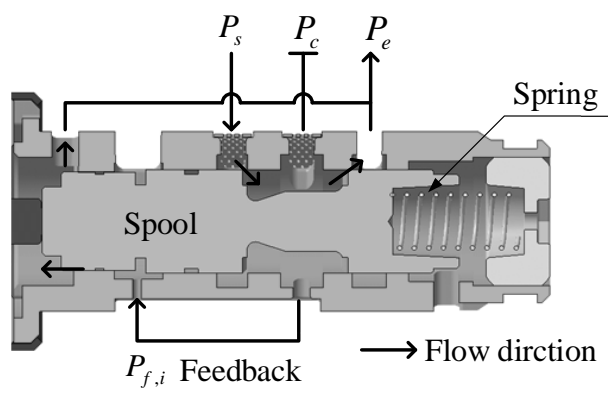

Fig. 2 The flow direction of a valve 
The far-field boundary condition is the magnetic insulation, i.e., the tangential component of the magnetic potential is zero. In the flow field, the boundary condition of the feedback pressure $P_{f, i}$ was to be equal to the control pressure and varied at every time step as shown in Fig. 2.

Fig. 3 shows the computational grid system for the linear control solenoid valve. The computational domain consists of three regions: the stationary zone, the deformed zone, and the moving zone. These zones are connected at the non-metering surface as shown in Fig. 3, b. To prevent the creation of an inverted mesh in the deformed zone, we used the Laplace smoothing method [18]. In order to describe the dynamic behavior of the system, the Arbitrary Lagrangian-Eulerian (ALE) method was employed [20]. The non-linear system solvers we used for the governing equations are the parallel sparse direct linear solvers developed by Olaf Schenk [21]. An implicit time-stepping technique was employed with a generalized- $\alpha$ method [22]. Further details of the computational algorithm and implementation are provided in References [18].

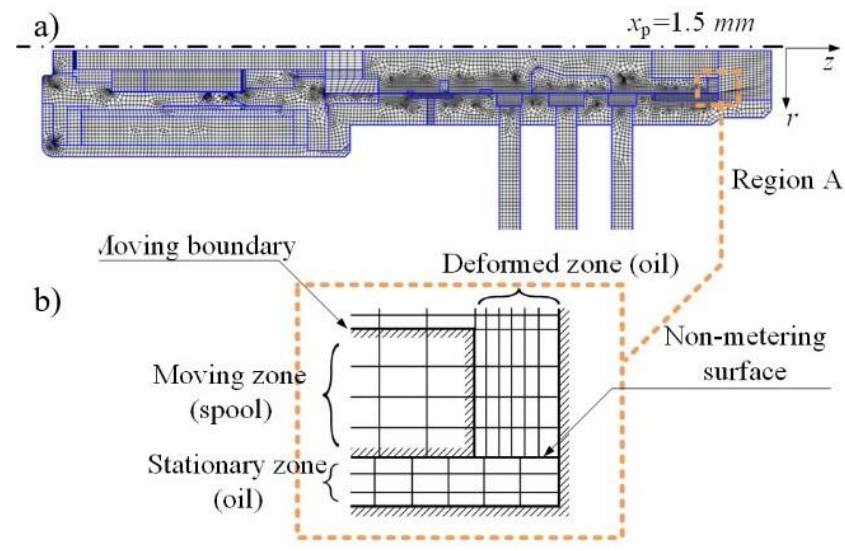

Fig. 3 Computational grids for the linear control solenoid valve: (a) overall mesh, (b) detailed moving mesh in region $\mathrm{A}$

\section{Results and discussion}

4.1. The geometrical redesign using the magnetostatic simulation

The geometry of the linear control solenoid valve is shown in Fig. 4. A typical solenoid valve has a nonlinear force-stroke curve [9]. As the stroke increases, the forces drastically increase due to the decrease in the main air gap $\delta$ as shown in Fig. 4, a. However, for linearity the electromagnetic force in the linear control solenoid valve must be constant with respect to variation in the plunger strokes. The electromagnetic forces are mainly affected by the shape of the core pole, the plunger, the yoke, and the number of coil turns. In particular, the conical shape of the core pole enables the maintenance of a constant electromagnetic force despite variation in the plunger stroke. The total magnetic flux through the plunger can be divided into the magnetic flux through the main air gap and the magnetic flux through the core cone [10]. Since the magnetic flux through the core cone does not affect the axial electromagnetic force, some amount of magnetic flux should be bypassed near of the minimum of $\delta$ from the geometrical redesign.

Thus, in order to improve the electromagnetic path for the electromagnetic component, the effect of the geometry of the core cone on the electromagnetic force was investigated via a magnetostatic analysis. The design parameters of the core cone are mainly the inner diameter $x_{1}$, the length of the core tip $x_{2}$, and the height of the core tip $x_{3}$. The design parameter of the hydraulic part is the port to port distance $L_{p}$. The design parameters of the linear control solenoid valve are summarized in Table 2.

Table 2

Design variables and their levels

\begin{tabular}{|c|c|c|c|}
\hline No. & Parameter & Unit & Levels \\
\hline 1 & $x_{1}$ & $\mathrm{~mm}$ & $7.5,8.5,9.5,10.5$ \\
\hline 2 & $x_{2}$ & $\mathrm{~mm}$ & $0.2,0.4,0.6,0.8$ \\
\hline 3 & $x_{3}$ & $\mathrm{~mm}$ & $\begin{array}{c}0.0,0.15,0.5, \\
0.75\end{array}$ \\
\hline 4 & $L_{p}$ & $\mathrm{~mm}$ & $11.2,11.3,11.5$ \\
\hline
\end{tabular}

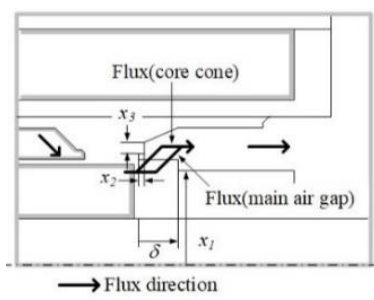

a)

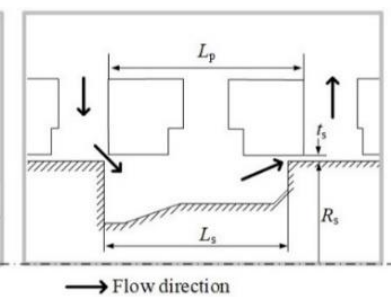

b)
Fig. 4 Geometry of the linear control solenoid valve: (a) electromagnetic component, (b) hydraulic component

The effects that varying the geometries of the core pole had on the electromagnetic forces in the magnetostatic simulations are shown in Fig. 5. The base model in Fig. 5 is the model proposed by Lee et al. [16]. In all simulations, the external current is $i=1.0 \mathrm{~A}$. When the inner diameter $x_{1}$ increases, the electromagnetic force decreases due to the increase of the reluctance at the main air gap, as shown in Fig. 5, a. The curve of the electromagnetic force culminates at $x_{p}=0.6 \mathrm{~mm}$ before gently falling. As the plunger stroke approaches the maximum $x_{p}=1.4 \mathrm{~mm}$, the electromagnetic forces steeply decrease because of the reduction of the amount of magnetic flux at the main air gap. The inner diameter of the base model is $10.5 \mathrm{~mm}$. Fig. 5, b represents the effects that varying the length of the core tip $x_{2}$ has on the electromagnetic forces. When the length of the core tip increases, the electromagnetic force decreases due to the increase of the bypass of magnetic flux from the plunger to the core cone. There is a marked decrease in the range of $x_{p}=0 \sim 0.3 \mathrm{~mm}$. The length of the base model's core tip is $0.4 \mathrm{~mm}$. The effects that varying the height of the core tip $x_{3}$ has on the electromagnetic forces are shown in Fig. 5, c. When the height of the core tip decreases, the angle of the core cone geometrically increases, as shown in Fig. 4, a. If the height of the core tip decreases, the electromagnetic force decreases in the range of $x_{p}=0 \sim 1.1 \mathrm{~mm}$ but increases in the range of $x_{p}=1.1 \sim 1.4 \mathrm{~mm}$. In the range of $x_{p}=$ $=0 \sim 1.1 \mathrm{~mm}$, the reluctance increases due to the magnetic 


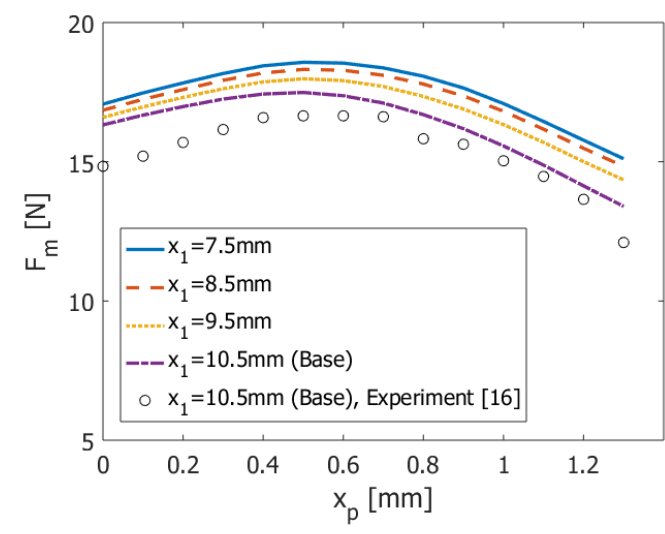

a)

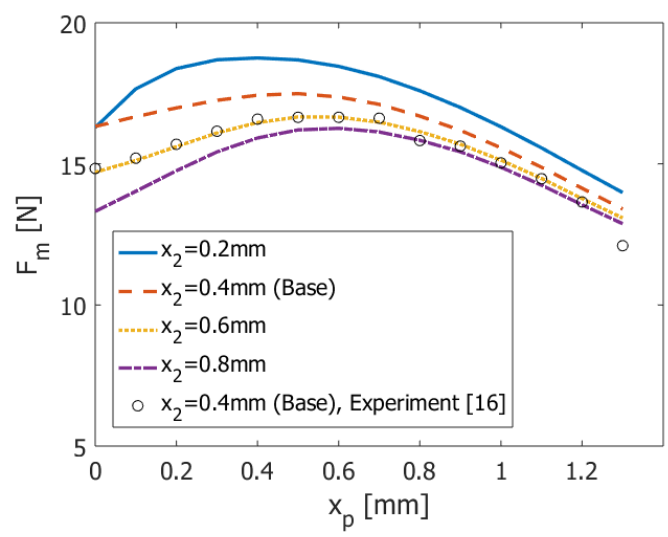

b)

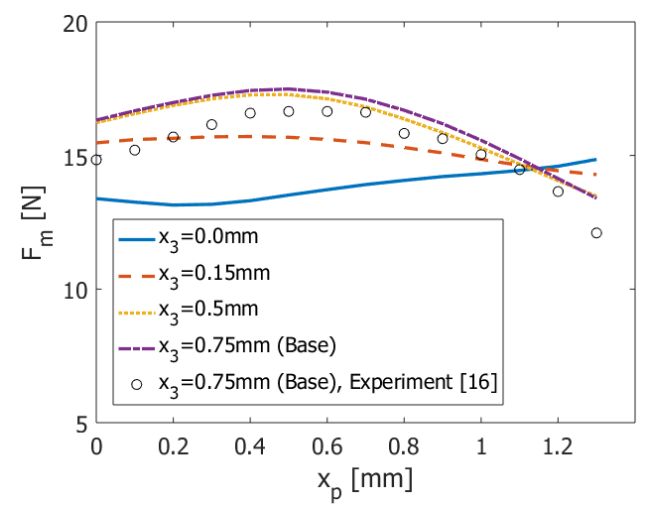

c)

Fig. 5 Effects of varying the geometries of the core cone on the electromagnetic forces in the magnetostatic simulations at $i=1.0 \mathrm{~A}$ : (a) core diameter $\left(x_{1}\right)$, (b) core tip length $\left(x_{2}\right),(\mathrm{c})$ core tip height $\left(x_{3}\right)$

saturation near the valve's core cone. Consequently, the electromagnetic force is reduced. On the other hand, in the range of $x_{p}=1.1 \sim 1.5 \mathrm{~mm}$, the magnetic flux at the main air gap increases due to the magnetic saturation near the conical cone of the core. The influence of the core tip's height on the variations of electromagnetic force mainly affect the plunger strokes. Using the results in Fig. 5, the redesign I model was proposed, in which the electromagnetic force was improved. Here, the inner diameter $x_{1}=0.95 \mathrm{~mm}$ of the core cone increases the electromagnetic force near the maximum stroke. The height of the core cone's core tip $x_{3}=$ $=0.15 \mathrm{~mm}$ reduces the deviation of the electromagnetic force with respect to the plunger strokes. The length of the core tip $x_{2}=0.4 \mathrm{~mm}$ is equal to that of the base model. The redesign I model's electromagnetic force average is $15.88 \mathrm{~N}$ and is similar to that of the base model, which was $16.045 \mathrm{~N}$. The redesign I model, which modified the geometry of the core cone of the base model, was able to obtain constant electromagnetic forces with respect to the plunger strokes.

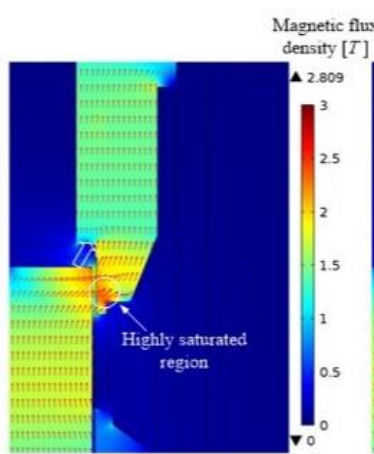

a)

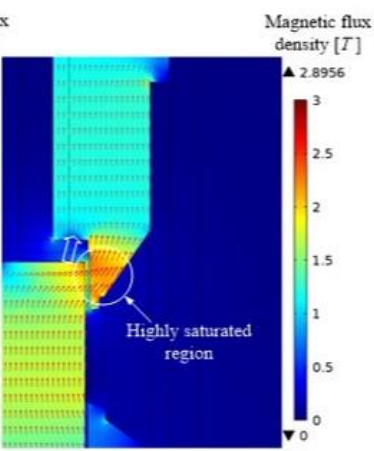

b)
Fig. 6 Magnetic flux density contours of varying the geometries of the core cone at $x_{p}=1.4 \mathrm{~mm}$ and $i=1.0 \mathrm{~A}$ : (a) the base model, (b) the redesign I model

Fig. 6 shows the magnetic flux density contours when varying the core pole geometries for the base model and the redesign I model at $x_{p}=1.4 \mathrm{~mm}$. The redesign I model's saturation area for the core cone is wider than that of the base model because $x_{2}=0.15 \mathrm{~mm}$. The magnetic flux which passes the main air gap increases because of the magnetic flux saturation area and the inner diameter $x_{1}=$ $=0.95 \mathrm{~mm}$. Thus, the electromagnetic force increases at $x_{p}=1.4 \mathrm{~mm}$. In addition, since the inner diameter of the core decreases, the reluctance at the main air gap is also reduced. The axial magnetic flux, which generates the electromagnetic force, increases. Consequently, the electromagnetic force is improved for the plunger strokes.

Fig. 7 depicts the electromagnetic force characteristics on the plunger strokes for various constant external currents, for the base model and for the redesign I model. The redesign I model's electromagnetic forces on the plunger strokes are more constant than those of the base model. The electromagnetic force of the redesign I model is similar to that of the base model in the range of $x_{p}=$ $=0 \sim 0.3 \mathrm{~mm}$ and is lower in the middle range $x_{p}=$ $=0.3 \sim 1.0 \mathrm{~mm}$. It is clear that in the range of $x_{p}=$ $=1.0 \sim 1.4 \mathrm{~mm}$, the electromagnetic force in the redesign I model has improved from that of the base model.

Fig. 8 represents the transient electromagnetic simulations coupled with a single DOF system for the varying geometries of the valve, for the base model and for redesign I model. The system parameters and the boundary conditions of the linear control solenoid valve are the same as those summarized in Table 2. The external currents when the spools begin to move are in all cases nearly identical to $i=0.75 \mathrm{~A}$, but the external currents are different when the spool approaches the maximum spool strokes. Consequently, the spool strokes depend on the external force variations. The spool stroke of the base model has a gentle slope 
beyond the external current $i=0.84 \mathrm{~A}$, where the electromagnetic force rapidly decreases, as shown in Fig. 7. Even if the external current increases, the spool strokes do not increase further.

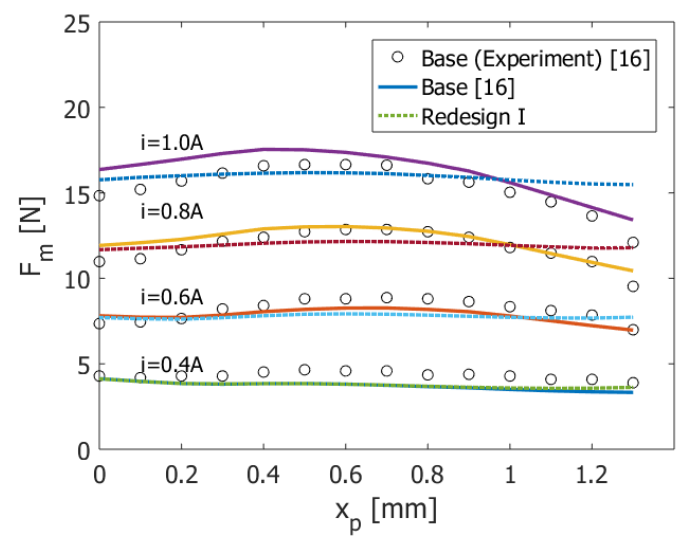

Fig. 7 Electromagnetic forces characteristics on the plunger strokes for various constant external currents

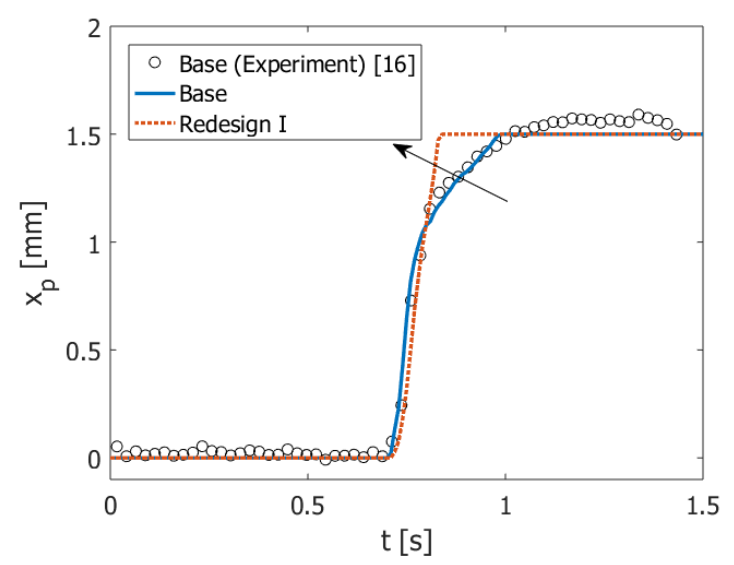

Fig. 8 The transient electromagnetic simulations coupled with a single DOF system for the varying the geometries of the valve, for the base model and for redesign I model

4.2. The geometrical redesign of the linear control solenoid valve using multiphyisics simulation

The multiphysics simulations were performed to investigate and analyze the effect of the system parameters, such as the spring constant $k_{s}$, the spring initial compression $x_{0}$, the number of coil turns $N_{p}$, and the geometrical redesign on the pressure-sensitivity curves of the valve. Even if the geometrical redesign of the core cone improves the linearity of the valve, the controllable range of the system remains narrow, as shown in Fig. 8. Without taking the hydraulic force into consideration, the controllable range of the redesign I model is $i=0.75 \sim 1.0 \mathrm{~A}, 20.8 \%$ of the total current range $i=0 \sim 1.2 \mathrm{~A}$. The hydraulic forces resulting from the feedback pressure enable a wider controllable range. Those forces, however, only reduce the current at which the spool begins to move. They do not affect the movement of the spool stroke beyond $x_{p}=1.1 \mathrm{~mm}$, when the hydraulic force approaches $0 \mathrm{~N}$. The geometrical redesign of the hydraulic component and the alteration of the system parameters are therefore needed in order to ensure the linearity of the liner control solenoid valve.
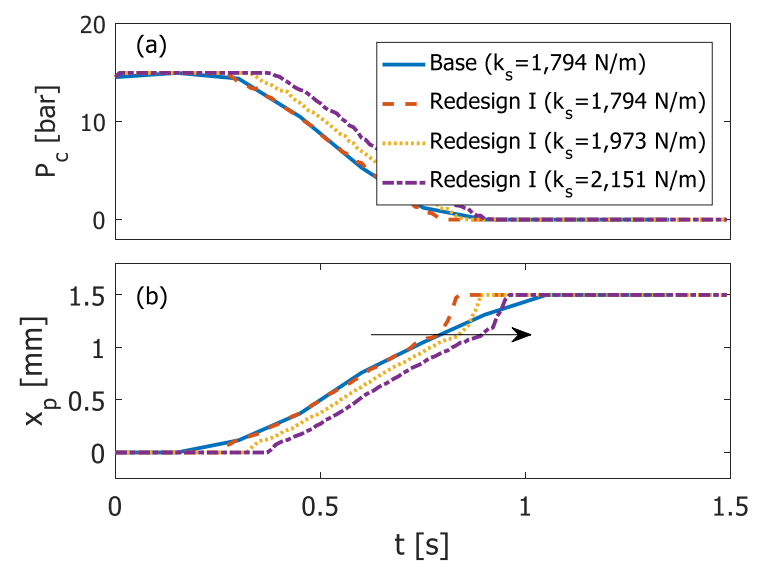

Fig. 9 Effects of varying the spring constants on the behaviors of the system using the multiphysics simulations: (a) control pressure, (b) spool stroke

The spring constant $k_{s}$ and the spring initial compression $x_{0}$ determine the magnitude of the restoring force in the system. Fig. 9 shows the spring constants of the valve on the dynamic behaviors of the system by using the multiphysics simulations. The spring constant of the base model is $1794 \mathrm{~N} / \mathrm{m}$. The hysteresis of the return spring is ignored in our simulations. In all cases, the hydraulic forces caused by feedback pressures broaden the controllable range of the spool stroke, as shown in Fig. 9, b. When the spring constant ranges from $1794 \mathrm{~N} / \mathrm{m}$ to $2152 \mathrm{~N} / \mathrm{m}$ in the redesign I model, the external current at which the spool begins to move is delayed from 0.18 A to $0.32 \mathrm{~A}$. As the spring constant increases, the reaction force also increases. Consequently, the pressure sensitivity curves are shifted forward at the same external current, as shown in Fig. 8, a. The spool stroke gradually increases until $x_{1}=1.1 \mathrm{~mm}$, and then its movement steeply increases, as shown in Fig. 8, b. The hydraulic force is $10.7 \mathrm{~N}$ at the supplied pressure of $15 \mathrm{bar}$, before slowly decreasing. After the spool stroke $x_{p}=1.1 \mathrm{~mm}$, the spool stroke's movement is determined by the relation between the electromagnetic and spring forces. The pressure sensitivity curves are also shifted forward at the same external current, as shown in Fig. 9, a.

The effects of varying the spring initial compressions on the system control characteristics by using multiphysics simulations are shown in Fig. 10. The spring initial compression of the base model is $6 \mathrm{~mm}$. The initial spring force $F_{s, 0}=k_{s} \times x_{\mathrm{o}}$ of the valve should be designed with a safety factor in mind, to prevent any undesirable valve movement caused by the vibration of the vehicle. When the initial compression of the return spring ranges from $6 \mathrm{~mm}$ to $8 \mathrm{~mm}$, the external current at which the spool begins to move ranges from $0.18 \mathrm{~A}$ to $0.4 \mathrm{~A}$. The effects of varying the spring initial compressions are similar to those resulting from the spring constants, as shown in Fig. 10, b. It is difficult to meet the target range of the pressure sensitivity curve.

Fig. 11 depicts the effects of varying the number of coil turns in the system control characteristics by using the multiphysics simulations. The base model's number of coil turns is 530. The number of coil turns is determined by the restricted coil resistance and the size of the bobbin, and it directly affects the electromagnetic force. As the number of 
coil turns increases, the out-of-plane component of the current density increases [16]. If the valve's number of coil turns is designed to be small, it can reduce power loss, which generates heat in the coil. When the number of coil turns decreases, the spool stroke is delayed at the given external current, as shown in Fig. 11, b. When, however, the spool begins to move, the current is nearly identical to $i=0.18 \mathrm{~A}$ when the number of coil turns is varied. At a low external current, there is little deviation of the electromagnetic force based on the number of coil turns.
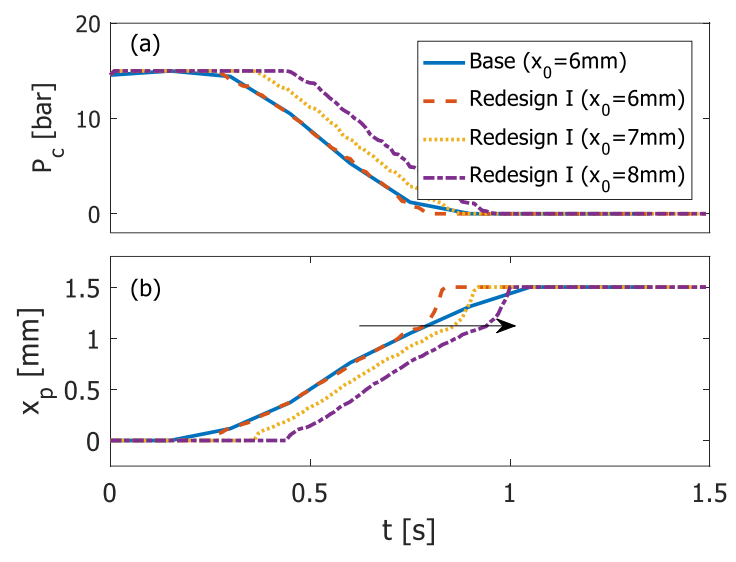

Fig. 10 Effects of varying the spring initial compressions on the behaviors of the system using the multiphysics simulations: (a) control pressure, (b) spool stroke
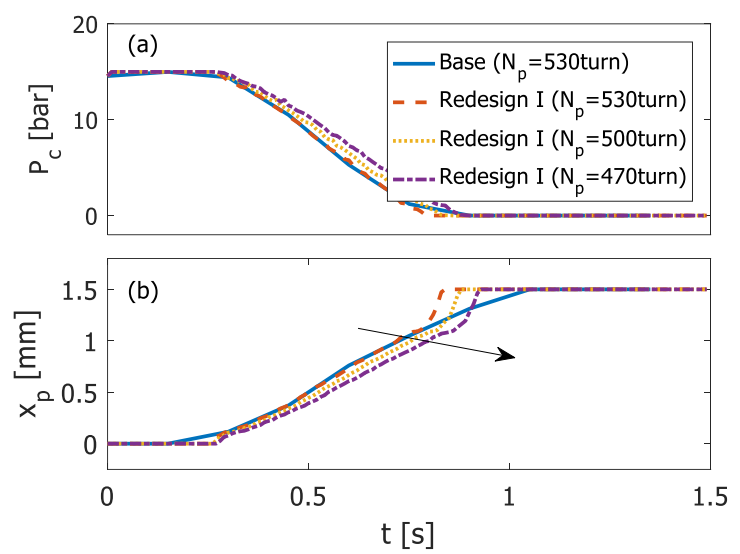

Fig. 11 Effects of varying the number of coil turns on the behaviors of the system using the multiphysics simulations: (a) control pressure, (b) spool stroke

The sudden increase of the spool stroke at $x_{p}=$ $=1.1 \mathrm{~mm}$ continues to appear in Figs. 9 to 11 . These results imply that the system parameters do not improve the linearity near $x_{p}=1.1 \mathrm{~mm}$. Because the control port is already open to the exhaust port at $x_{p}=1.1 \mathrm{~mm}$, the hydraulic force cannot move the spool in a forward direction. To obtain the linearity of the valve, the hydraulic force should be maintained to a maximum spool stroke $x_{p}=1.5 \mathrm{~mm}$. The effects of the geometrical redesign of the valve's hydraulic component are shown in Fig. 12. As the port-to-port distance $L_{p}$ of the sleeve increases, the spool stroke is increasingly linear, as shown in Fig. 12, b. When the port-to-port distance is $11.5 \mathrm{~mm}$, the spool stroke's linearity is in the controllable range $i=0.18 \sim 0.9 \mathrm{~A}$; the inflection point of $x_{p}=1.1 \mathrm{~mm}$ disappears. The redesign of the hydraulic component can affect the behavior of the valve for the spool stroke's whole range.
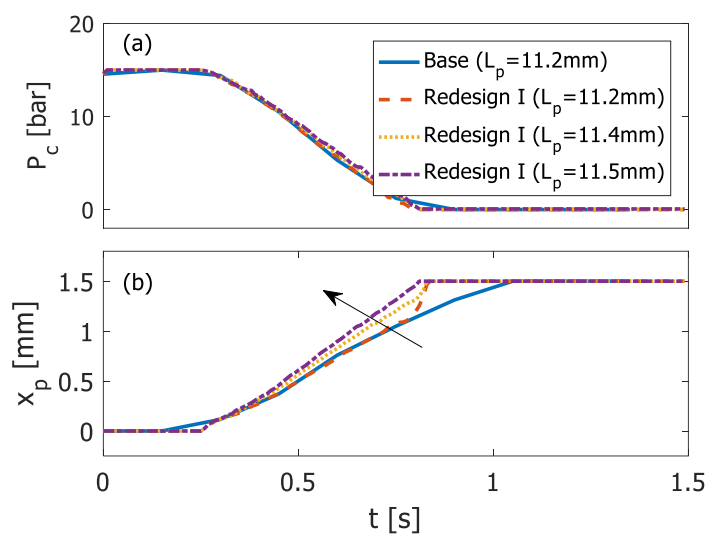

Fig. 12 Effects of varying the port to port distance on the behaviors of the system using the multiphysics simulations: (a) control pressure, (b) spool stroke
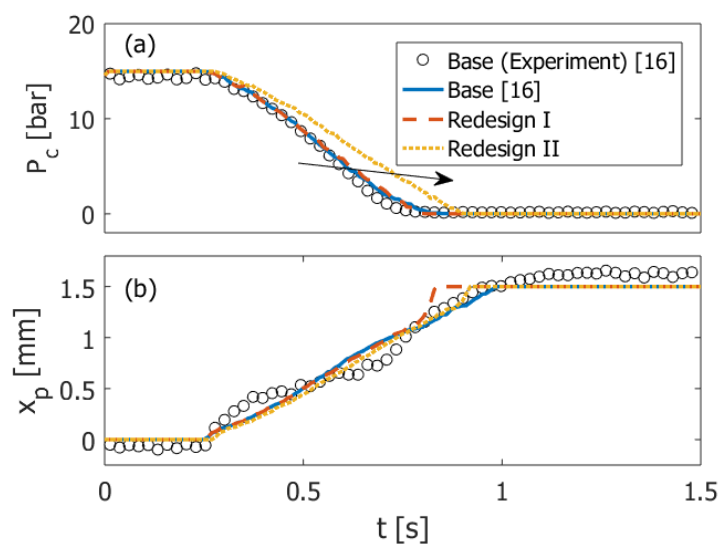

Fig. 13 Effects of the geometrical redesign on the behaviors of the system using the multiphysics simulations: (a) control pressure, (b) pool stroke

Table 3

Geometrical redesign of a linear control solenoid valve

\begin{tabular}{|c|c|c|c|c|}
\hline $\begin{array}{c}\text { Param- } \\
\text { eter }\end{array}$ & Unit & $\begin{array}{c}\text { The base } \\
\text { model }\end{array}$ & $\begin{array}{c}\text { The rede- } \\
\text { sign I model }\end{array}$ & $\begin{array}{c}\text { The redesign } \\
\text { II model }\end{array}$ \\
\hline$x_{1}$ & $\mathrm{~mm}$ & 10.5 & 9.5 & 9.5 \\
\hline$x_{2}$ & $\mathrm{~mm}$ & 0.4 & 0.4 & 0.4 \\
\hline$x_{3}$ & $\mathrm{~mm}$ & 0.75 & 0.15 & 0.15 \\
\hline$L_{p}$ & $\mathrm{~mm}$ & 11.2 & 11.2 & 11.5 \\
\hline$k_{S}$ & $\mathrm{~N} / \mathrm{m}$ & 1,974 & 1,974 & 1,974 \\
\hline$x_{0}$ & $\mathrm{~mm}$ & 6 & 6 & 6 \\
\hline$N_{p}$ & turns & 530 & 530 & 470 \\
\hline
\end{tabular}

In this study, the controllable external current is $i=0 \sim 1.2$ A for the linear control solenoid valve. However, with the inclusion of the hydraulic forces, the control pressure of the base model was controlled in the range of $i=0.18 \sim 0.84 \mathrm{~A}$; at $55 \%$ of the valve's total control range. The effective range for the pressure control should be widened to $i=0.18 \sim 1.0$ A for the geometrical redesign of the valve. Using the results of Figs. 9 to 12, we employed the redesign II model, which uses the redesign I model for its 
electromagnetic component, $L_{p}=11.5 \mathrm{~mm}$ for its hydraulic component, and $N_{p}=470$ turns for its system parameter. The geometry and the system parameter are summarized in Table 3.

Fig. 13 shows the results of the dynamic behaviors of the base model, the redesign I model, and the redesign II model using the multiphysics simulations. The geometrical redesign of the hydraulic component $L_{p}=11.5 \mathrm{~mm}$ maintains the feedback pressure until $x_{p}=1.5 \mathrm{~mm}$. The number of coil turns $N_{p}=470$ turns delays the arrival point of the spool stroke in the forward direction. By having $N_{p}=470$ turns, the redesign II model can linearly control the spool stroke in the range of $i=0.18 \sim 1.0 \mathrm{~A}$. Due to the geometrical redesign of the hydraulic component $L_{p}=11.5 \mathrm{~mm}$, the hydraulic force moves the spool in the forward direction, as shown in Fig. 13, b. The behavior of the redesign model II's spool stroke is similar to that of the base model, but the control pressure characteristics are different, as shown in Fig. 13, a. This result implies that the redesign II model, which employed the geometrical improvements of the core cone and port-to-port distance, can accurately control the pressure for the external current $i=0.18 \sim 1.0 \mathrm{~A}$.

\section{Conclusions}

The geometrical redesign was conducted to improve the performance of the linear control solenoid valve using a multiphysics simulation coupled with a single DOF system. Although the hydraulic feedback force broadens the controllable pressure range of the linear control solenoid valve, the range of the spool stroke for the pressure control is still narrow at $i=0.18 \sim 0.84 \mathrm{~A}$. The redesign I model, which modifies the geometry of the core cone, obtains constant electromagnetic forces with respect to the plunger strokes. We employed the redesign II model, which has improved the electromagnetic and hydraulic components. The behavior of this model's spool stroke, with $N_{p}=470$ turns, is similar to that of the base model, but the pressure sensitivity curves are highly improved. This result entails that the redesign II model can accurately control the pressure while the external current is $i=0.18 \sim 1.0 \mathrm{~A}$, given that it was at $68.3 \%$ of the total control range.

\section{Acknowledgment}

This work is supported by a 2017 research grant from Youngsan University, Republic of Korea.

\section{References}

1. Merritt, H. E. 1967. Hydraulic Control Systems, John Wiley \& Sons, New York.

2. Brejcha, M. F. 1982. Automatic Transmissions, Prentice-Hall, Englewood Cliffs.

3. Davis, J. A.; Stewart, M. 2002. Predicting Globe Control Valve Performance-Part I: CFD Modeling, ASME Journal of Fluids Engineering 124: 772-777. http://dx.doi:10.1115/1.1490108.

4. Davis, J. A.; Stewart, M. 2002. Predicting Globe Control Valve Performance-Part II: Experimental Verification, ASME Journal of Fluids Engineering 124(3): 778-
783.

http://dx. doi:10.1115/1.1490126.

5. Yuan, Q.; Li, P. Y. 2005. Using Steady Flow Force for Unstable Valve Design: Modeling and Experiments, ASME Journal of Dynamic Systems, Measurement, and Control 127: 451-462. http://dx.doi:10.1115/1.1997166.

6. Lee, G. S.; Sung, H. J.; Kim, H. C.; Lee, H. W. 2010. Flow Force Analysis of a Variable Force Solenoid Valve for Automatic Transmissions, ASME Journal of Fluids Engineering 132: 031103-1-7. http://dx. doi:10.1115/1.4001070.

7. Amirante, R.; Moscatelli, P. G.; Catalano, L. A. 2007. Evaluation of the Flow Forces on a Direct (Single Stage) Proportional Valve by Means of a Computational Fluid Dynamic Analysis, Energy Conversion and Management 48: 942-953. https://doi.org/10.1016/j.enconman.2006.08.024.

8. Xie, Z. 2011. Effects of Flow Forces on a Flow Control Variable Force Solenoid, SAE paper 2011-01-0394. http://dx.doi: 10.4271/2011-01-0394.

9. Lequesne, B. P. 1998. Finite Element Analysis of a Constant-Force Solenoid for Fluid Flow Control, IEEE, Transaction Industrial Application 24(4): 574-581. https://doi.org/10.1109/28.6107.

10. Arakawa, T.; Niimi, S. 2002. Optimization Technology of Magnetic Circuit for Linear Solenoid, SAE Paper 2002-01-0565. http://dx.doi: 10.4271/2002-01-0565.

11. Vaughan, N. D.; Gamble, J. B. 1996. The Modeling and Simulation of a Proportional Solenoid Valve, ASME Journal of Dynamic Systems, Measurement, and Control 118(1): 120-125 http://dx.doi:10.1115/1.2801131.

12. Pappalardo, C. M.; Guida, D. 2017. Adjoint-based Optimization Procedure for Active Vibration Control of Nonlinear Mechanical Systems, ASME Journal of Dynamic Systems, Measurement, and Control 139(8): 081010.

http://dx.doi: 10.1115/1.4035609.

13. Watanabe, H.; Ichise, S.; Nagaoka, T.; Tsuchiya, T. 2005. Development of Compact and High Performance Fuel Injector using Electromagnetic Field Simulation, SAE paper 2005-32-0019.

14. Bircher, F.; Marmet, P. 2009. Multiphysics Modeling of a Micro Valve, Proceedings of the European COMSOL conference, Paper No. 6679, Milan, Italy.

15. Naseradinmousavi, P.; Nataraj, C. 2011. Nonlinear Mathematical Modeling of Butterfly Valves Driven by Solenoid Actuators, Applied Mathematical Modelling 35: 2324-2335. https://doi.org/10.1016/j.apm.2010.11.036.

16. Lee, G. S.; Sung, H. J.; Kim, H. C. 2012. Multiphysics Analysis of a Linear Control Solenoid Valve, Journal of Fluids Engineering-Trans ASME 135: 011104. http://dx.doi: 10.1115/1.4023079.

17. Mutschler, K.; Dwivedi, S.; Kartmann, S.; Bammesberger, S.; Koltay, P.; Zengerle, R.; Tanguy, L. 2014. Multi Physics Network Simulation of a Solenoid Dispensing Valve, Mechatronics 24: 209-221. https://doi.org/10.1016/j.mechatronics.2014.02.005.

18. COMSOL. 2016. COMSOL Multiphysics 5.0 User's Guide. 
19. Jin, J. 2002. The Finite Element Method in Electromagnetics, 2nd edition, Wiley-IEEE Press.

20. Hirt, C. W.; Amsden, A. A.; Cook, J. L. 1997. An Arbitrary Lagrangian-Eulerian Computing Method for all Flow Speeds, Journal of computer physics 135: 198-216. http://www.pardiso-project.org/. https://doi.org/10.1006/jcph.1997.5702.

21. Jansen, K. E.; Whiting, C. H.; Hulbert, G. M. 2000. A Generalized- $\alpha$ Method for Integrating the Filtered Navier-Stokes Equations with a Stabilized Finite Element Method, Computer Methods in Applied Mechanics and Engineering, 190, 305-319.

https://doi.org/10.1016/S0045-7825(00)00203-6.
Gee-Soo Lee

\section{DESIGN IMPROVEMENT OF A LINEAR CONTROL SOLENOID VALVE USING MULTIPHYSICS SIMULATION}

S u m m a r y

Multiphysics simulation coupled with a 1-DOF (Degree of Freedom) system was carried out to improve the geometrical redesign of the linear control solenoid valve. Since the electromagnetic field is strongly coupled with the flow field, a multiphysics simulation coupled with a 1-DOF system could offer a considerably useful approach in the design and optimization of linear control solenoid valves. First, in order to maintain a constant electromagnetic force on plunger strokes with an external current flow, the electromagnetic component valve was improved through a magnetostaic simulation. Although the hydraulic feedback force widened the controllable pressure range of the linear control solenoid valve, the range of the spool stroke for the pressure control was still narrow for $i=0.18 \sim 0.84 \mathrm{~A}$. We employed the redesign II model, which improved the electromagnetic and the hydraulic components. The redesign II model was able to accurately control the pressure while the external current was $i=0.18 \sim 1.0 \mathrm{~A}$, at $68.3 \%$ of the total control range.

Keywords: multiphysics simulation, linear control solenoid valve, linearity.

Received 'February 10, 2018

Accepted June 14, 2018 